\title{
Analysis of Real-Time Estimation Method Based on Hidden Markov Models for Battery System States of Health
}

\author{
Changhao Piao ${ }^{*}$, Zuncheng $\mathrm{Li}^{*}$, Sheng Lu* ${ }^{*}$ Zhekui Jin*, and Chongdu Cho ${ }^{\dagger}$ \\ *Institute of Pattern Recognition and Applications, Chongqing University of Posts and Telecommunications, \\ Chongqing, China \\ †Department of Mechanical Engineering, Inha University, Incheon, Korea
}

\begin{abstract}
A new method is proposed based on a hidden Markov model (HMM) to estimate and analyze battery states of health. Battery system health states are defined according to the relationship between internal resistance and lifetime of cells. The source data (terminal voltages and currents) can be obtained from vehicular battery models. A characteristic value extraction method is proposed for HMM. A recognition framework and testing datasets are built to test the estimation rates of different states. Test results show that the estimation rates achieved based on this method are above $90 \%$ under single conditions. The method achieves the same results under hybrid conditions. We can also use the HMMs that correspond to hybrid conditions to estimate the states under a single condition. Therefore, this method can achieve the purpose of the study in estimating battery life states. Only voltage and current are used in this method, thereby establishing its simplicity compared with other methods. The batteries can also be tested online, and the method can be used for online prediction.
\end{abstract}

Key words: Characteristic value, Health states, Hidden Markov model, Internal resistance

\section{INTRODUCTION}

The electric vehicle (EV) has become the main research focus of the automobile industry with the development of economies and the concomitant lack of energy sources [1]. A power battery, which is one of the core blocks of an EV, has been influencing the development of the automobile industry. Thus, competition in the EV industry is reduced to competition in power battery technologies. EVs will be widely developed only when mature technology, low cost, and high safety of power batteries have been attained. Therefore, current studies on power battery performance status have become popular in many countries [2].

Lifetime is a key performance index with regard to battery technology [3], [4]. At present, the development of power

Manuscript received Mar. 24, 2015; accepted Jun. 30, 2015

Recommended for publication by Associate Editor Jonghoon Kim.

†Corresponding Author: cdcho@inha.ac.kr

Tel: +82-32-860-7300, Fax: +82-32-868-1716, Inha University

* Institute of Pattern Recognition and Applications, Chongqing University of Posts and Telecommunications, China battery technologies is in a stagnant stage. Studies on battery life prediction have a significant value for power battery systems in several areas, such as effective management, improvement of vehicle dynamic performance, and safety and economic performance. Battery life can be evaluated by examining cell capacity and internal resistance. This study proposes a method to study battery health states. The assessment is divided into four states according to the differences in battery resistance.

Thus far, many researchers have focused on the design of battery mechanism models. Researchers must build efficient battery models to simulate real batteries. Some of these models have elicited good performances [5]-[9]. However, they require calculations of many parameters. Battery parameters are always nonlinear, thereby complicating the calculations [10], [11]. Data-driven means have shown a great potential in solving the aforementioned problems, and they have led to significant progress [12]-[16]. Complex relationships among electrochemical parameters are not considered by these methods, which can analyze problems from the gathered data. 
In [13], the authors proposed a method to describe the battery capacity under particular conditions in which battery capacity testing took considerable amounts of time. Similarly, a significant amount of time is required to test several cycles in [12], in which an artificial neural network (ANN) method of testing cycles was proposed.

The hidden Markov model (HMM) is a data-driven method. Given the complicated configuration parameters for battery simulation models and the accuracy of HMM in estimating results, this study proposes a method of battery life state estimation through an HMM. On the basis of the changes in battery data, we preprocess these data and propose a method to obtain effective character values for the HMM. Subsequently, an HMM framework is built to estimate battery health state. Finally, test data are collected to generate testing estimation results.

\section{HIDDEN MARKOV MODEL}

An HMM includes a dual stochastic process [17]. The first is a Markov chain, which describes state transitions. The other is a stochastic process, which describes the statistical relationships between states and observational variables. An HMM can be usually described as the function $\lambda=(\pi, A, B)$, where $\pi$ is the initial probability of each state, $A=\left(a_{i j}\right)_{N \times N}$ is a state transition matrix, $a_{i j}$ describes the probability of state $i$ transferring to state $j, N$ is the number of states, and $B$ is the probability distribution of observations for each state. $B$ can be described by several Gaussian distributions, which are written as

$$
\begin{gathered}
b_{j}\left(o_{t}\right)=\sum_{\ell=1}^{M} c_{j \ell} N\left(o_{t} \mid \mu_{j \ell}, \Sigma_{j \ell}\right) \\
N\left(o_{t} \mid \mu_{j l}, \Sigma_{j l}\right)=\frac{1}{(2 \pi)^{d / 2}\left|\Sigma_{j l}\right|^{1 / 2}} e^{-\frac{1}{2}\left(x-\mu_{j l}\right)^{\mathrm{T} \Sigma_{j l}^{-1}\left(x-\mu_{j l}\right)}}
\end{gathered}
$$

where $b_{j}\left(o_{t}\right)$ is the probability of observation $o_{t}$ in state $j$, $M$ is the number of Gaussian distributions, $\mu_{j l}$ and $\Sigma_{j l}$ are the mean and covariance values of the Gaussian density, and $c_{j l}$ is the weight of the $l$ th Gaussian component in state $j$. Therefore, an HMM can be also described as $\lambda=\left(\pi, A, \mu_{j l}, \Sigma_{j l}, c_{j l}\right)$, which is used in this paper.

Given an HMM, the following three essential problems must be solved to make this HMM usable in practical applications [18]:

1) Probability calculation: Given some observation sequences $O=\left\{o_{1}, o_{2}, \cdots, o_{T}\right\}$ and $\lambda$, where $T$ is the length of the sequence, to calculate $P(O \mid \lambda)$, which are the probabilities of $O$ occurring under condition $\lambda$, a forward-backward algorithm can be used to resolve this problem.
2) Decoding: Given specific $O$ sequence and condition $\lambda$, the best state sequence $Q=\left\{q_{1}, q_{2}, \cdots, q_{T}\right\}$ explaining $O$ is determined. Viterbi [19] or forward-backward algorithms can solve this problem.

3) Training: The parameters of $\lambda$ are reevaluated until $P(O \mid \lambda)$ increases to definitive rules. Baum-Welch algorithm solves this problem.

\section{DATA ANALYSIS}

Battery health states can be described with internal resistance or capacity. A large resistance or low capacity represents a short lifetime. However, an accurate capacity cannot be obtained easily. Therefore, based on resistance, battery lifetime is divided into four states in this study, namely, state 1 , state 2 , state 3 , and state 4 . This partition method is shown in [17], which classifies the states of the bearings based on fault diameters. State 1 represents the initial resistance. States 2 and 3 are middle resistance states. State 4 is the failure-state resistance. The internal resistance increases from state 1 to state 4 sequentially.

Terminal voltage and charging-discharging current obviously change in a battery system. These two parameters can be obtained easily, and they are two of the main variables that are monitored by the battery management system. The outward manifestation of a battery state change can be described with voltage and current. Therefore, terminal voltage and charging-discharging current serve as the source data for this study.

The battery system of a vehicle model proposed in [11] is applied to our research to obtain battery data. Standard velocity conditions, which can enhance the reliability of generated data, are included in the vehicle model. Four conditions are used in this study, namely, ECE + EUDC, FTP72, 10-15, and CCDC, which refer to European, American, Japanese, and Chinese conditions, respectively. All of them are typical conditions and used frequently, as shown in Fig. 1. These conditions are associated with various changes, such as constant velocity and variable speed. Therefore, the data have various forms that benefit the HMMs.

The battery system of the vehicle model has 50 lithium ion cells. The four internal resistances are $0.285,0.57,0.855$, and $1.14 \Omega$, which map to states $1,2,3$, and 4 , respectively. If one of the resistances is kept constant, then the currents can be estimated according to vehicle speeds. Therefore, terminal voltage can be obtained based on the internal resistance and current.

As shown in Fig. 1, the four conditions have different times. The sample time of this vehicle model is $6 \times 10^{-5} \mathrm{~s}$. The sample interval is set to 400 to obtain sufficient data. In other words, the sampling time is $24 \mathrm{~ms}$. An initial dataset is also generated. As shown in Figs. 2, 3, 4, and 5, the horizontal axis 

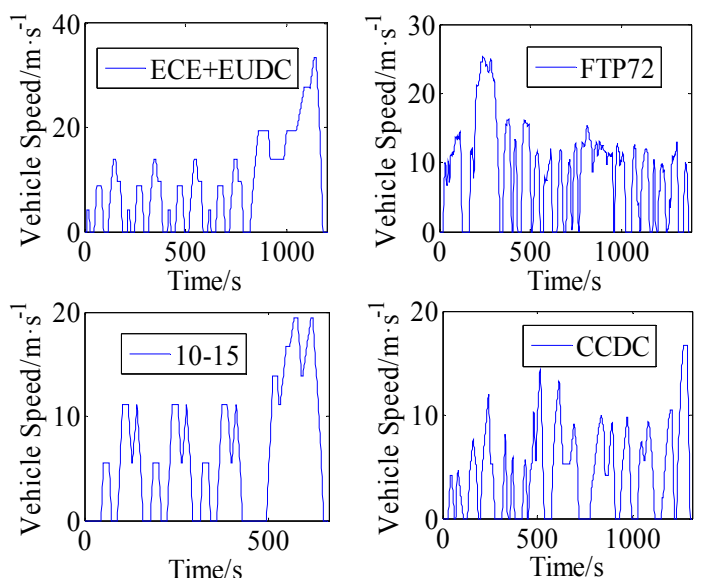

Fig. 1. Vehicular speed variation with time under four different vehicle driving conditions.
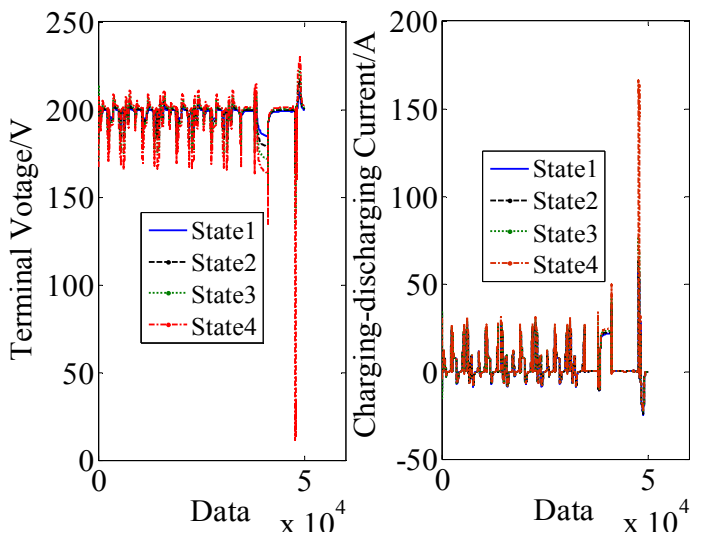

Fig. 2. Battery data for the four states under ECE + EUDC condition.
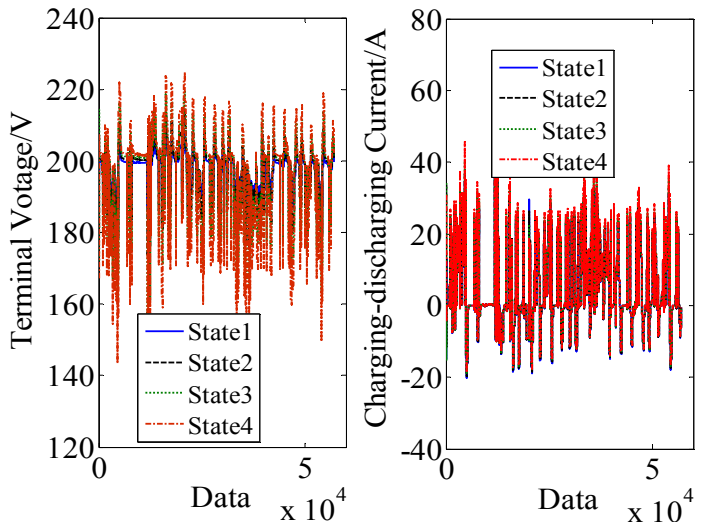

Fig. 3. Battery data for the four states under FTP72 condition.

shows the data sample number. Different data quantities under the four conditions exist because of the different time conditions. Positive currents indicate that batteries are discharging, whereas negative currents indicate that batteries are charging.

A statistical method shown in [20] is used to verify the reliability of the gathered data by calculating internal resistances. Given the effect of the current dispersion, the allowed error of statistical method is $15 \%$. The currents that are
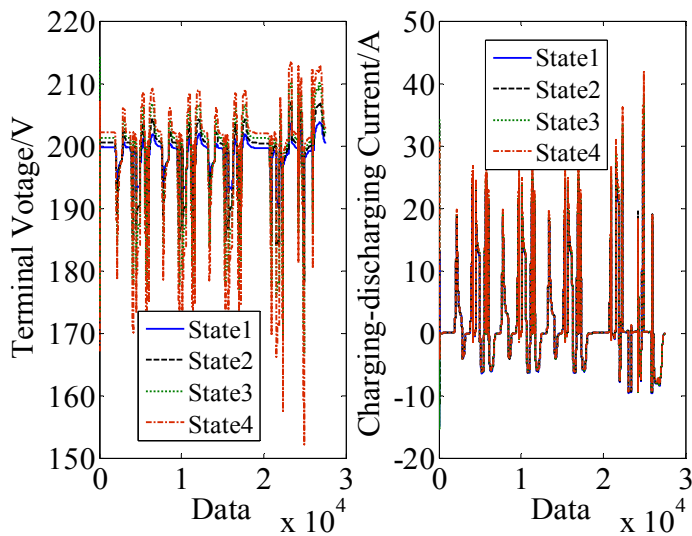

Fig. 4. Battery data for the four states under 10-15 condition.
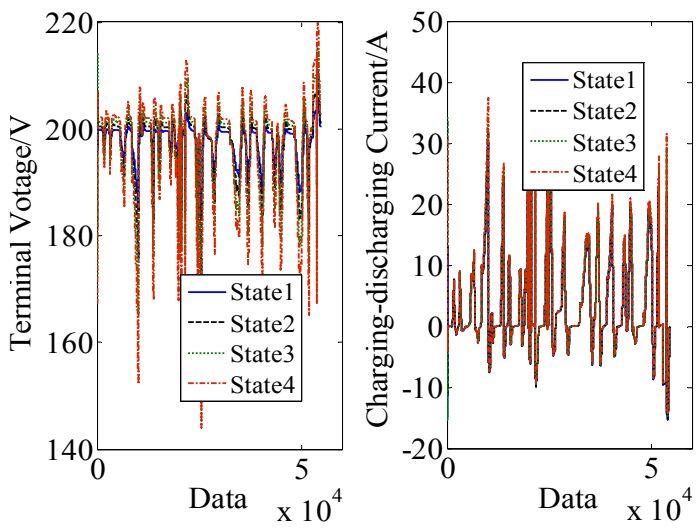

Fig. 5. Battery data for the four states under CCDC condition.

TABLE I

Statistical Method for Calculating InTERnal Resistances

\begin{tabular}{|c|c|c|c|}
\hline Condition & $\begin{array}{l}\text { Internal } \\
\text { resistance in } \\
\text { battery system } \\
(\Omega)\end{array}$ & $\begin{array}{l}\text { Resistances } \\
\text { obtained with } \\
\text { the statistical } \\
\text { method }(\Omega)\end{array}$ & $\begin{array}{l}\text { Relative } \\
\text { error } \\
(\%)\end{array}$ \\
\hline \multirow{4}{*}{$\begin{array}{l}\mathrm{ECE}+ \\
\text { EUDC }\end{array}$} & 0.285 & 0.278 & 2.4 \\
\hline & 0.57 & 0.581 & 1.9 \\
\hline & 0.855 & 0.832 & 2.7 \\
\hline & 1.14 & 1.145 & 0.4 \\
\hline \multirow{4}{*}{ FTP72 } & 0.285 & 0.294 & 3.2 \\
\hline & 0.57 & 0.583 & 2.3 \\
\hline & 0.855 & 0.880 & 2.9 \\
\hline & 1.14 & 1.163 & 2.0 \\
\hline \multirow{4}{*}{$10-15$} & 0.285 & 0.290 & 1.8 \\
\hline & 0.57 & 0.567 & 0.5 \\
\hline & 0.855 & 0.859 & 0.5 \\
\hline & 1.14 & 1.172 & 2.8 \\
\hline \multirow{4}{*}{ CCDC } & 0.285 & 0.297 & 4.2 \\
\hline & 0.57 & 0.595 & 4.4 \\
\hline & 0.855 & 0.884 & 3.4 \\
\hline & 1.14 & 1.166 & 2.3 \\
\hline
\end{tabular}

extremely close are removed in this study. The statistical results are shown in Table I.

Table I shows that the relative errors of statistical resistance are all less than 5\%, which is within the allowable scope. This calculation method verifies the reliability of the data. 


\section{HMM-BASED ESTIMATION}

\section{A. Estimation Framework}

As shown in Fig. 6(a), voltages and currents are gathered from the vehicle model. After obtaining these characteristic values through the proposed extraction method, all models are acquired through HMM training. For this training, Baum-Welch and forward-backward algorithms can be used. Correspondingly, $\lambda_{1}, \lambda_{2}, \lambda_{3}, \lambda_{4}$ are the training results for the four HMMs. Fig. 6(b) shows the collection of the test data that occurs first. After the feature vectors are obtained from test data, probabilities are calculated with forward or backward algorithms according to the HMMs. Finally, the maximum probability values are estimated as the result. The states that respond to the maximum probability values are the recognition results.

\section{B. Feature Extraction}

Efficient feature parameter sequences, which are used to train HMMs, can improve estimation rates. The characteristic values of the data must be extracted before the HMM training. Figs. 2, 3, 4, and 5 illustrate that for the four states at each condition, many charging-discharging currents with similar magnitudes exist, whereas terminal voltages (U) obviously change. According to the change of the data, we preprocess the data by dividing the voltages by the currents (U/I). Therefore, the dataset is composed of $U$ and $U / I$ values. After the preprocessing, the change of the data is apparent in the time domain.

First, eight parameters (i.e., mean-squared value, effective value, mean value, standard deviation, dispersion coefficient, median, skewness coefficient, and kurtosis coefficient) have been calculated as features, and some parameters change inconspicuously. The mean-square value, effective value, mean value, and median of $U$ clearly change. A normalization method is used to solve this problem because some values are large, whereas some are small. All the voltages are positive. Thus, a max-normalization method can be used to normalize the mean-square value, effective value, mean value, and median of U, as shown in Equ. (3). For U/I, its mean value and its dispersion coefficient apparently change. Given that the currents attain negative values, we can normalize them between $[-1,1]$, as shown in Equ. (4).

$$
\begin{gathered}
X^{*}=\frac{X}{\operatorname{Max}}, \\
X^{*}=\frac{2 *(X-\text { Min })}{\text { Max }- \text { Min }}-1,
\end{gathered}
$$

where $X$ is the set of characteristic values before normalization, and $X^{*}$ is the set of characteristic values after normalization. Max is the maximum value of the characteristic values, and Min is the minimum of the characteristic values.

Therefore, after normalizing the characteristic values, they

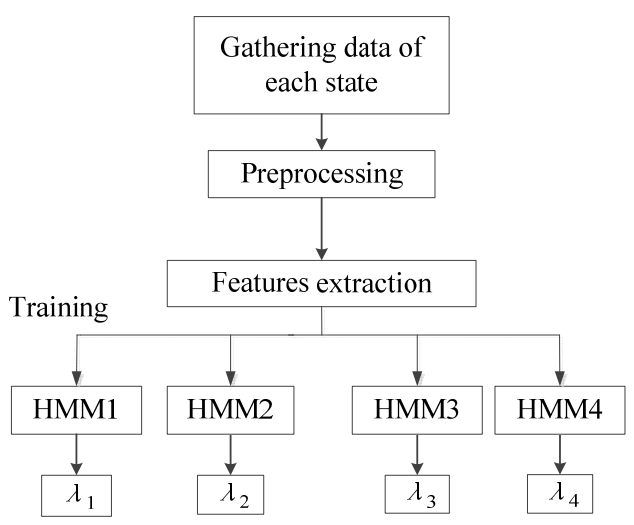

(a)

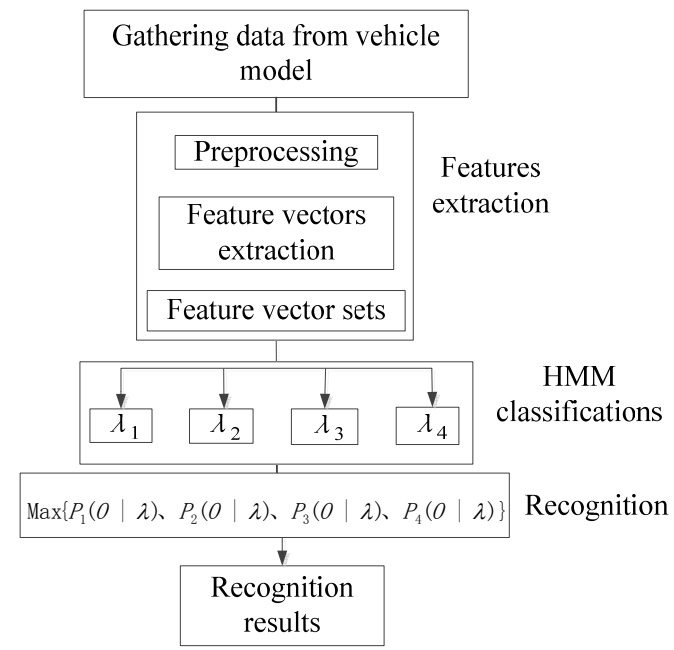

(b)

Fig. 6. HMM-based estimation framework.

can be regarded as observations for HMM training. Fig. 7 illustrates that for ECE + EUDC condition, every 500 points are treated as a frame, and 100 frames exist altogether. In Fig. 7 , the numbers of feature vectors are plotted as the abscissa values and the characteristic values as the ordinate values.

We do not discuss the characteristic extraction of the other three conditions in detail because the extraction method is the same. The characteristic values can be calculated in accordance with Equs. (5)-(11) as follows:

Mean-square of U: $\frac{\sum_{k=1}^{S} y_{U}(k)^{2}}{S}$

Effective value of $\mathrm{U}: \frac{\operatorname{Max}\left(y_{U}(k)\right)}{\sqrt{2}}$

Mean value of $\mathrm{U}: \frac{\sum_{k=1}^{S} y_{U}(k)}{S}$

Median of U:

$$
\frac{\left(y_{U}(S / 2)+y_{U}(S / 2+1)\right)}{2}, S \text { for even number }
$$



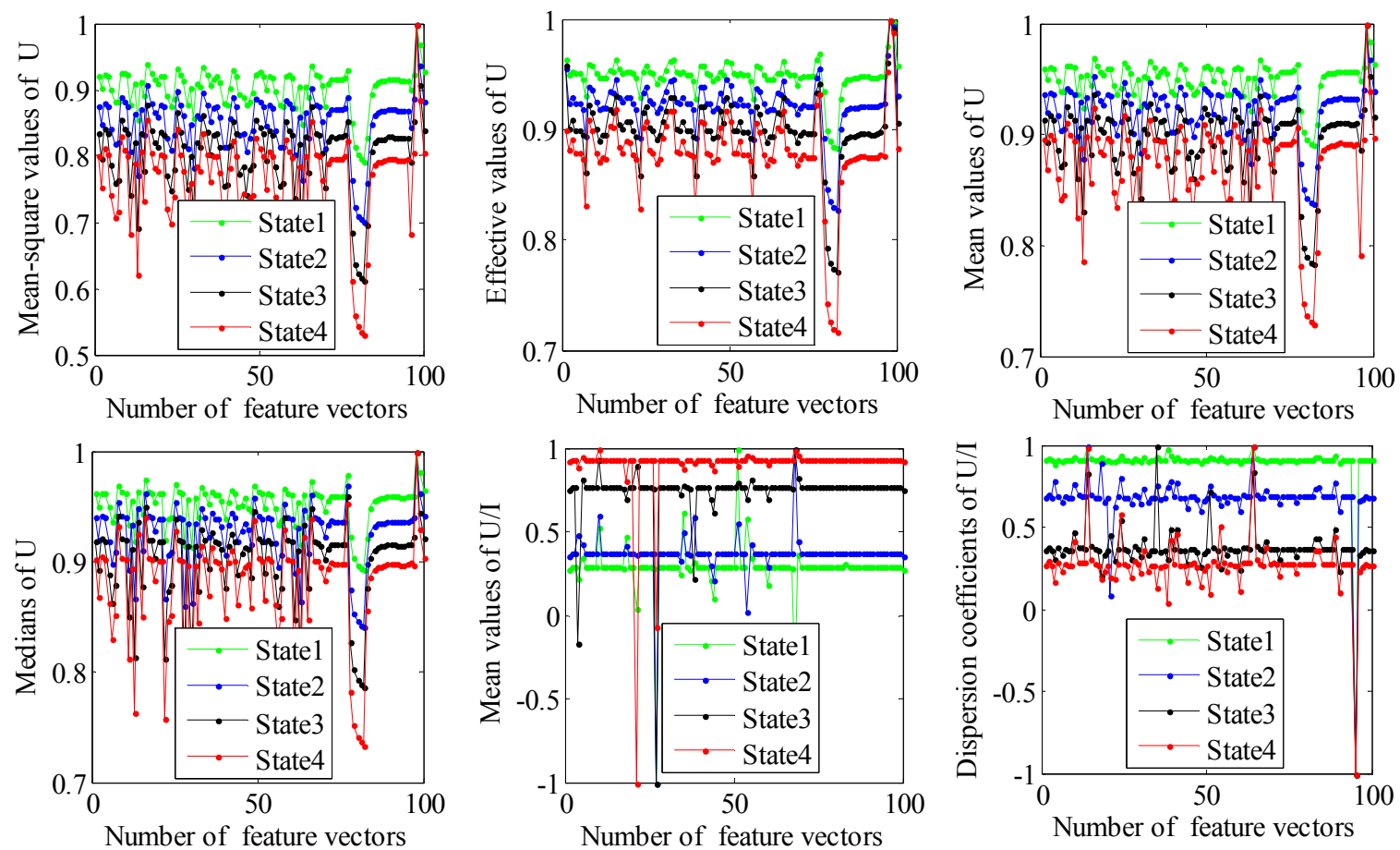

Fig. 7. Feature vectors under ECE + EUDC condition.

$\frac{y_{U}(S / 2+1)}{2}, S$ for odd number

Mean value of U/I: $\frac{\sum_{k=1}^{S} y_{U / I}(k)}{S}$, written for $\overline{y_{U / I}}$

Dispersion coefficient of U/I: $\sqrt{\frac{\sum_{k=1}^{S}\left(y_{U / I}(k)-\overline{y_{U / I}}\right)^{2} / S}{\overline{y_{U / I}}}}$

\section{Estimation of Battery States of Health}

The internal resistances show an increasing trend in the process of using our battery system. The battery system health states change sequentially from state 1 to state 4 . We can set $\pi=[1,0,0,0]^{\mathrm{T}}$ when we initialize the HMM parameters. The transition matrix can be set as

$$
A=\left[\begin{array}{cccc}
0.5 & 0.5 & 0 & 0 \\
0 & 0.5 & 0.5 & 0 \\
0 & 0 & 0.5 & 0.5 \\
0 & 0 & 0 & 1
\end{array}\right] .
$$

Thus, the present state can transfer only to itself or to the state behind it, as shown in Fig. 8 .

Fig. 7 illustrates that the changes of the characteristic values do not conform to normal distributions. However, a mixture of several Gaussian distributions can be used to describe the probabilities of the characteristic values, as indicated in Eqs. (1) and (2). For this study, we set $M=3$. In addition, $\mu_{j l}, \Sigma_{j l}$, and $c_{j l}$ can be calculated with a k-means

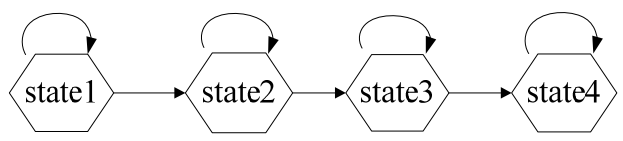

Fig. 8. Four-state left-to-right HMM.

clustering algorithm based on the given data. The algorithm is not discussed comprehensively in this paper. The error precision of $\mathrm{k}$-means is set at 0.0001 .

In general, four states for each condition exist, and each state is composed of three Gaussian components. Every Gaussian component has a single mean $\mu$. Therefore, 12 mean values $\mu$ must be initialized for the four states. The calculation steps are as follows:

1) From 100 observations, 12 vectors are randomly selected as the initial clustering centers, in which we can obtain 12 classes.

2) The Euclidean distance between observations and centers is calculated. Each observation has 12 distance values. According to the minimum value, every observation can be a response to a class.

3) The mean of every class, which we can obtain from Step 2, is calculated. Then, we can obtain 12 new centers.

4) The new centers are used instead of the centers from Step 2. Steps 2 and 3 are repeated.

5) If errors meet the requirement, then the calculation is discontinued.

6) The 12 new centers, which serve as the 12 mean values, are outputted. 
According to Equ. (12), the covariances of the Gaussian densities can be obtained with

$$
\Sigma_{j}=\frac{\sum_{i=1}^{D_{j}}\left(o_{j i}-\mu_{j}\right)}{D_{j}}, 1 \leq \mathrm{j} \leq 12,
$$

where $\sum_{j}$ is the covariance of the $j$ th class, $D_{j}$ is the number of observations for the $j$ th class, and $o_{j i}$ and $\mu_{j}$ are the observations and the mean for the $j$ th class, respectively. Then, 12 mean values and 12 covariance values are obtained. The reshape function is then used to change $\mu$ or $\sum$ into a $4 \times 3$ matrix with responses to the four states and the three Gaussian components. Given that each observation consists of six characteristic values, $\mu$ becomes a $6 \times 4 \times 3$ matrix, and $\sum$ becomes a $6 \times 6 \times 4 \times 3$ matrix. Three positive numbers with a sum of 1 are generated randomly as the weights because each state has three Gaussian components. This case is the same for the other states.

After initialization, some characteristic value sequences are used to train HMM for a state. Baum-Welch algorithm can be used to train an HMM. The training stops when the number of iterations is less than 40 or the convergence error is less than 0.0001 .

Fig. 9 shows the process of estimation by comparing forward probabilities. After training, the characteristic values can be extracted from the test data. According to the training results, forward probabilities can be calculated based on HMM parameters. Calculation starts from the first group of feature vectors. $T$ is the number of groups in total. After the forward probabilities are calculated, the corresponding state of the maximum probability is the estimation result. The next group of feature vectors is then estimated.

\section{EXPERIMENTS}

As mentioned earlier, the data were obtained from a vehicle model that has several typical conditions. The experiments were conducted under ECE + EUDC, FTP72, 10-15, and CCDC conditions. The condition times were 1200 , 1372, 661, and $1314 \mathrm{~s}$. We kept the internal resistance of each state constant. We then gathered data once every $24 \mathrm{~ms}$, after leading in the initial data.

Our experiments were conducted with MATLAB. After collecting the source data, one characteristic value could be obtained for every 500 data points. As mentioned in the previous section, trained HMMs could be acquired. An HMM under one condition might not be able to estimate the state of another condition with satisfactory performance because HMMs were different under different conditions. Therefore, we estimated the states not only under one condition but also under a hybrid condition. All the data were then combined in

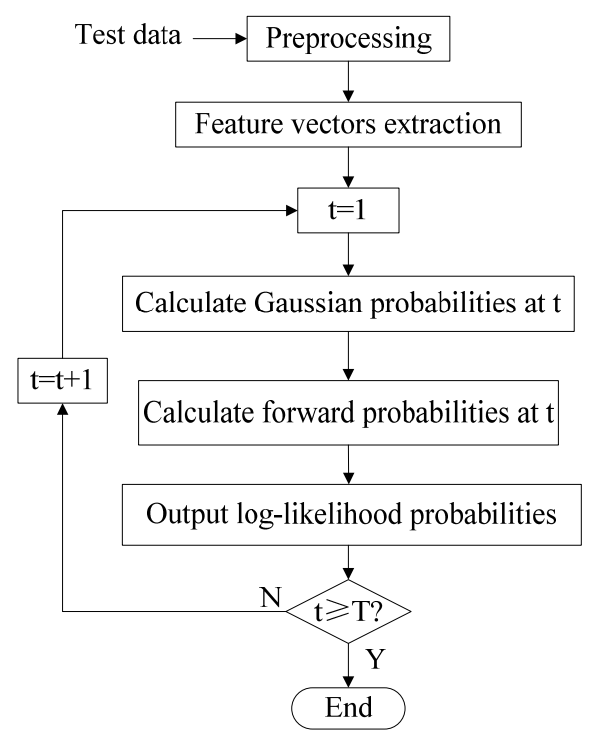

Fig. 9. Process of calculating probabilities for test data.
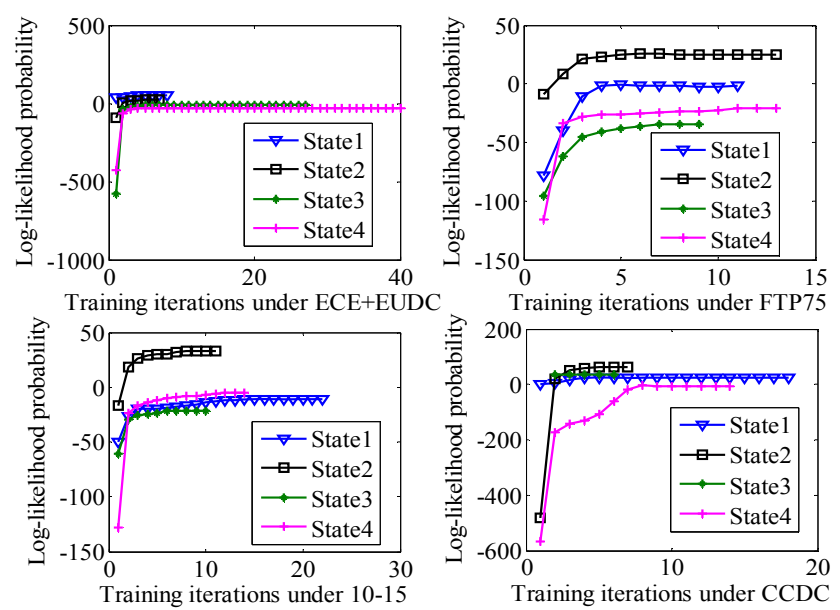

Fig. 10. Log-likelihoods of HMMs during training under the four conditions.

an order according to the state. For example, the data of states $1,2,3$, and 4 included the data of states 1, 2, 3, and 4, respectively, under all conditions. If an HMM was trained with the new data, then the HMM could be used to estimate the states under different conditions.

Figs. 10 and 11 illustrate that for the condition with the convergence error, the numbers of iterations were all less than 40. Finally, the probabilities tended to attain invariant values. This finding indicated that an HMM accomplished its training objective.

After training, the parameters of each HMM could be obtained. The training results of state 1 for the condition of FTP72 are given as follows:

Initial probabilities: $\pi=[1,0,0,0]^{\mathrm{T}}$,

$$
\text { Transition matrix: } A=\left[\begin{array}{cccc}
0.9848 & 0.0135 & 0 & 0 \\
0 & 0.9663 & 0.0337 & 0 \\
0 & 0 & 0.8623 & 0.1377 \\
0 & 0 & 0 & 1
\end{array}\right] \text {, }
$$




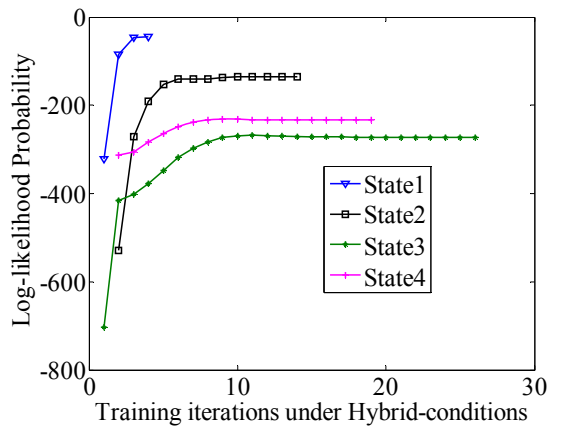

Fig. 11. Log-likelihoods of HMMs during training under hybrid conditions.

Means of Gaussians: $A=\left[\begin{array}{lll}0.9304 & 0.9249 & 0.9304 \\ 0.9650 & 0.9629 & 0.9650 \\ 0.9644 & 0.9616 & 0.9644 \\ 0.9610 & 0.9631 & 0.9609 \\ 0.7562 & 0.7524 & 0.7562 \\ 0.6359 & 0.3949 & 0.6357\end{array}\right]$,

Gaussian covariance:

$$
\begin{aligned}
\Sigma_{11} & =\left[\begin{array}{llllll}
0.0112 & 0.0005 & 0.0006 & 0.0007 & 0.0001 & -0.0005 \\
0.0005 & 0.0102 & 0.0002 & 0.0002 & 0 & -0.0002 \\
0.0006 & 0.0002 & 0.0103 & 0.0004 & 0 & -0.0002 \\
0.0007 & 0.0002 & 0.0004 & 0.0105 & 0 & -0.0003 \\
0.0001 & 0 & 0 & 0 & 0.0114 & 0.0008 \\
-0.0005 & -0.0002 & -0.0002 & -0.0003 & 0.0008 & 0.0184
\end{array}\right], \\
\Sigma_{12} & =\left[\begin{array}{llllll}
0.0108 & 0.0003 & 0.0004 & 0.0004 & 0 & 0.0019 \\
0.0003 & 0.0101 & 0.0001 & 0.0001 & 0 & 0.0004 \\
0.0004 & 0.0001 & 0.0102 & 0.0002 & 0 & 0.0010 \\
0.0004 & 0.0001 & 0.0002 & 0.0103 & 0 & -0.0003 \\
0 & 0 & 0 & 0 & 0.0105 & 0.0014 \\
0.0019 & 0.0004 & 0.0010 & -0.0003 & 0.0014 & 0.0753
\end{array}\right], \\
\sum_{13} & =\left[\begin{array}{llllll}
0.0112 & 0.0005 & 0.0006 & 0.0007 & 0.0001 & -0.0005 \\
0.0005 & 0.0102 & 0.0002 & 0.0002 & 0 & -0.0002 \\
0.0006 & 0.0002 & 0.0103 & 0.0004 & 0 & -0.0002 \\
0.0007 & 0.0002 & 0.0004 & 0.0105 & 0 & -0.0003 \\
0.0001 & 0 & 0 & 0 & 0.0114 & 0.0007 \\
-0.0005 & -0.0002 & -0.0002 & -0.0003 & 0.0007 & 0.0182
\end{array}\right],
\end{aligned}
$$

$$
\text { Weights of Gaussian: } c=\left[\begin{array}{lll}
0.4436 & 0.0811 & 0.4753 \\
0.3161 & 0.0675 & 0.6165 \\
0.0001 & 0.6981 & 0.3018 \\
0.3413 & 0.0027 & 0.6560
\end{array}\right],
$$

where $\mu$ represents the mean of the three Gaussian components for state 1 , and $\sum_{i j}$ represents the covariance of the $j$ th component for the $i$ th state.

$$
\begin{aligned}
B_{1}= & c_{11} \frac{1}{(2 \pi)^{d / 2} \sqrt{\left|\sum_{11}\right|}} e^{-\frac{1}{2}\left(O_{t}-\mu_{1}\right)^{\mathrm{T}} \Sigma_{11}{ }^{-1}\left(O_{t}-\mu_{1}\right)}+ \\
& c_{12} \frac{1}{(2 \pi)^{d / 2} \sqrt{\left|\sum_{12}\right|}} e^{-\frac{1}{2}\left(O_{t}-\mu_{2}\right)^{\mathrm{T}} \Sigma_{12}{ }^{-1}\left(O_{t}-\mu_{2}\right)}+ \\
& c_{13} \frac{1}{(2 \pi)^{d / 2} \sqrt{\left|\sum_{13}\right|}} e^{-\frac{1}{2}\left(O_{t}-\mu_{3}\right)^{\mathrm{T}} \sum_{13}{ }^{-1}\left(O_{t}-\mu_{3}\right)}
\end{aligned}
$$

According to Equs. (15)-(19), the probability for state 1 could be obtained in accordance to Equ. (20), where $B_{1}$ represents the probability of $O_{t}$ for state 1 , and $c_{11}, c_{12}$, and $c_{13}$ are the first, second, and third columns of the first row of $c$ in Equ. (19), respectively. $\mu_{1}, \mu_{2}$, and $\mu_{3}$ are the first, second, and third columns of $\mu$ in Equ. (15), respectively. $O_{t}$ represents an observation, and $d$ is the dimension of $O_{t}$, which is equal to 6 in this study. Thus, $B_{2}, B_{3}$, and $B_{4}$ could be obtained based on Equs. (1) and (2).

After obtaining $B$ and the HMM parameters, a forward algorithm can be used to calculate $P(O \mid \lambda)$ as follows:

Input: model $\lambda_{i}$, the probabilities $B_{i}\left(O_{t}\right)$ for observation $O$ at the $i$ th state.

Output: $P\left(O_{t} \mid \lambda_{i}\right)$

$$
\begin{aligned}
& \text { 1: } \alpha_{i}(1)=\pi_{i} B_{i}\left(O_{1}\right) \\
& \text { 2: } \alpha_{j}(t+1)=\left[\sum_{i=1}^{N} \alpha_{i}(t) a_{i j}\right] B_{j}\left(O_{t+1}\right), 1 \leq \mathrm{t} \leq \mathrm{T}-1 \\
& \text { 3: } \quad P\left(O_{t+1} \mid \lambda_{i}\right)=\sum_{i=1}^{N} \alpha_{i}(t+1), 1 \leq \mathrm{t} \leq \mathrm{T}-1
\end{aligned}
$$

where $\alpha_{i}(t)=P\left(O_{1}, O_{2}, \cdots, O_{t}, Q_{t}=i \mid \lambda\right)$ is the probability of the sequence $O_{1}, O_{2}, \cdots, O_{t}$ that ends up in state $i, Q_{t}$ represents the state of $O_{t}, a_{i j}$ is the probability of state $i$ transferring to state $j$, and $N$ represents the number of states at each condition.

The results were divided into two groups to test the estimation results: one group was under a single condition, whereas the other was under hybrid conditions. A part of the gathered data were selected randomly and regarded as the testing data for each state. Thus, repetition of testing and training data could be avoided. After extracting the characteristic values of the data, these values could be introduced into HMM to calculate the forward probabilities. Fig. 12 shows the estimation results of state 1 under ECE + EUDC condition. Fig. 12 indicates that 35000 data existed, namely, 70 groups of characteristic values. Most log-likelihood probabilities of state 1 were larger than those of the other three states. Only two probabilities did not belong to state 1 . Table II indicates that the estimation rate was $97.1 \%$. Fig. 13 shows the estimation results of state 4 for FTP72 condition. The estimation rates are shown in Table II.

Table II indicate that all the estimation rates were greater than $90 \%$. The rates could not achieve $100 \%$ because some data of different states were similar, thereby making the characteristic values similar.

The data from a mixture of states under single and hybrid conditions were constructed to test our results further. The hybrid conditions were according to the order of ECE + EUDC, FTP72, 10-15, and CCDC. Fig. 14 shows the 


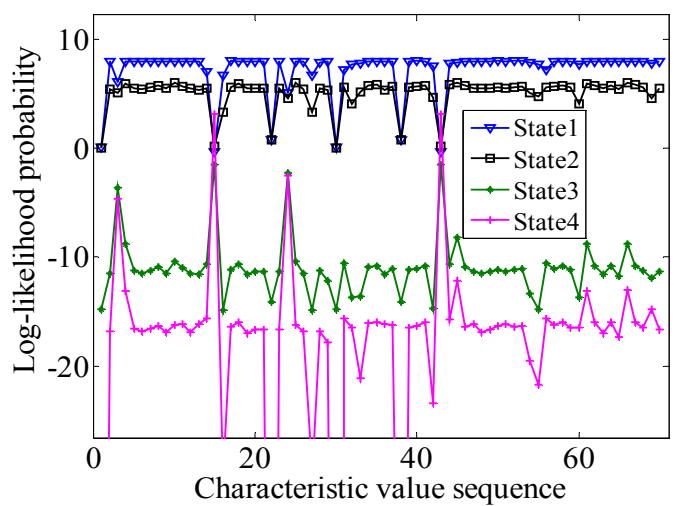

Fig. 12. Estimation results of state 1 under the ECE + EUDC condition.

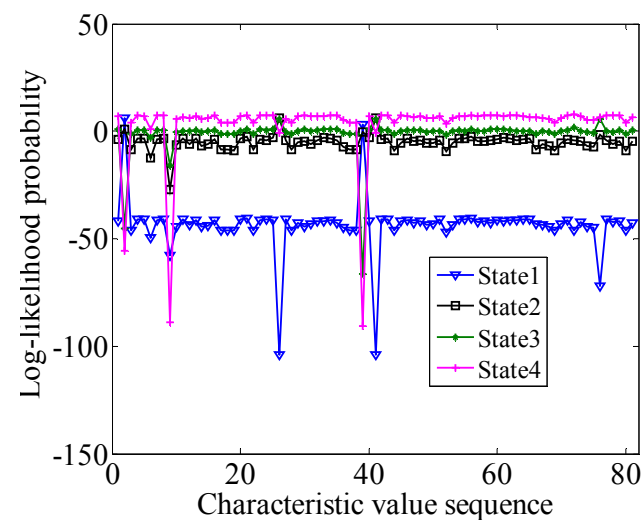

Fig. 13. Estimation results of state 4 under FTP72 condition.

TABLE II

ESTIMATION RESULTS FOR BATTERY SYSTEM STATES UNDER A SINGLE CONDITION

\begin{tabular}{ccccccc}
\hline Condition & States & $\lambda_{1}$ & $\lambda_{\mathbf{2}}$ & $\lambda_{\mathbf{3}}$ & $\boldsymbol{\lambda}_{\mathbf{4}}$ & $\begin{array}{c}\text { Estimation } \\
\text { rate (\%) }\end{array}$ \\
\hline \multirow{5}{*}{ ECE + EUDC } & $\mathrm{r}_{1}$ & 68 & 0 & 2 & 0 & 97.1 \\
& $\mathrm{r}_{2}$ & 3 & 46 & 0 & 0 & 93.9 \\
& $\mathrm{r}_{3}$ & 0 & 0 & 38 & 2 & 95.0 \\
& $\mathrm{r}_{4}$ & 1 & 0 & 1 & 71 & 97.3 \\
\hline \multirow{5}{*}{ FTP72 } & $\mathrm{r}_{1}$ & 43 & 0 & 1 & 0 & 97.7 \\
& $\mathrm{r}_{2}$ & 1 & 57 & 1 & 0 & 96.6 \\
& $\mathrm{r}_{3}$ & 2 & 2 & 78 & 0 & 95.1 \\
& $\mathrm{r}_{4}$ & 2 & 2 & 1 & 76 & 93.8 \\
\hline \multirow{4}{*}{$10-15$} & $\mathrm{r}_{1}$ & 46 & 1 & 1 & 1 & 93.9 \\
& $\mathrm{r}_{2}$ & 0 & 40 & 3 & 0 & 93.0 \\
& $\mathrm{r}_{3}$ & 0 & 0 & 35 & 1 & 97.2 \\
& $\mathrm{r}_{4}$ & 0 & 1 & 0 & 33 & 97.1 \\
\hline \multirow{5}{*}{ CCDC } & $\mathrm{r}_{1}$ & 79 & 7 & 0 & 0 & 91.9 \\
& $\mathrm{r}_{2}$ & 2 & 52 & 1 & 0 & 94.5 \\
& $\mathrm{r}_{3}$ & 2 & 1 & 64 & 2 & 92.8 \\
& $\mathrm{r}_{4}$ & 2 & 1 & 1 & 50 & 92.6 \\
\hline \multirow{6}{*}{} & & & & & &
\end{tabular}

estimation result for the mixture states under 10-15 condition. These test data included 11500 data of state 1, 14500 data of state 2, 14000 data of state 3 , and 18000 data of state 4 . The data were arranged in order from state 1 to state 4 . The observation sequence consisted of 23 groups of state 1, 29

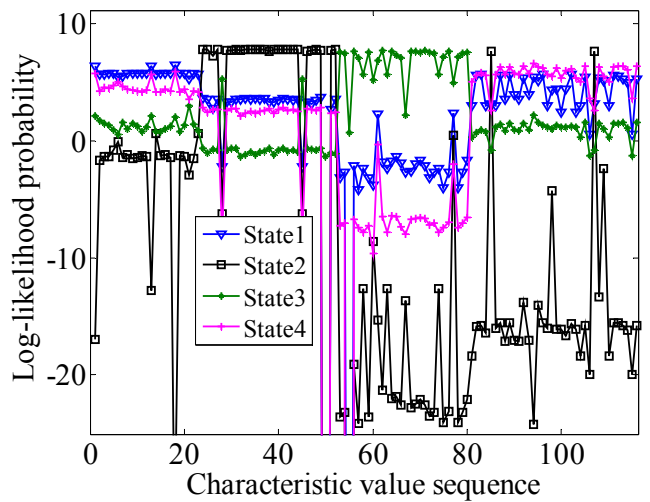

Fig. 14. Estimation results of mixture states under 10-15 condition.

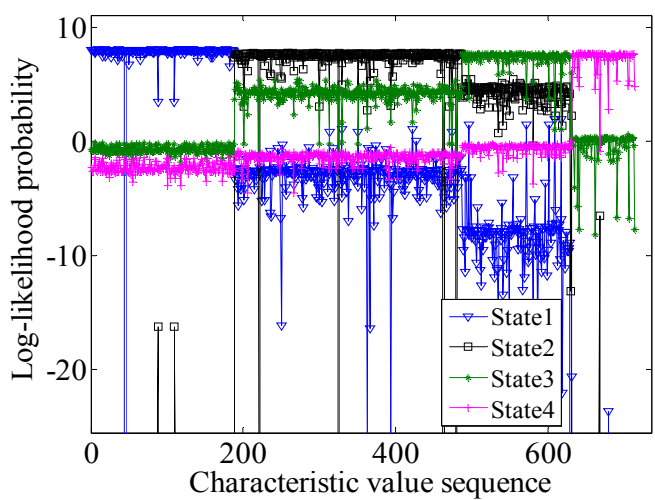

Fig. 15. Estimation results of mixture states under hybrid condition.

groups of state 2, 28 groups of state 3 , and 36 groups of state 4. In general, nearly all the observations corresponded to the matching states except for the five observations. Fig. 15 shows the estimation result for mixture states under hybrid condition. The statistical results of the mixture states under single and hybrid conditions are shown in Table III.

As illustrated in Table III, all the data were selected randomly. The values below the column labels $\lambda_{1}, \lambda_{2}, \lambda_{3}$, and $\lambda_{4}$ are the numbers of correctly estimated results. The estimation rates of mixture states under single and hybrid conditions could achieve values above $90 \%$. Meanwhile, we could use the HMMs that correspond to hybrid conditions to estimate the states that were under single conditions.

ANN is the most frequently used among the available study methods of battery health states [21]. All other factors being equal, this study used BP-ANN to estimate the states. The outputs of BP-ANN were the internal resistances that respond to the states. The inputs were the feature vectors extracted by the aformentioned method. The training data of BP-ANN were the same as the data of HMM under hybrid conditions, and so are the test data. Fig. 16 shows the results estimated with BP-ANN. The estimation results were proved correct when the estimation and actual values were equal. Based on the analyses, 535 sequences were estimated correctly in the entire set of 714 sequences. Therefore, the estimation rate was approximately $74.9 \%$. The proposed 
TABLE III

Estimation Results of MiXture States under Single And Hybrid Conditions

\begin{tabular}{|c|c|c|c|c|c|c|c|c|c|}
\hline \multirow{2}{*}{ Condition } & \multicolumn{4}{|c|}{ Mixture State } & \multirow{2}{*}{$\lambda_{1}$} & \multirow{2}{*}{$\lambda_{2}$} & \multirow{2}{*}{$\lambda_{3}$} & \multirow{2}{*}{$\lambda_{4}$} & \multirow{2}{*}{ Rate $(\%)$} \\
\hline & $r_{1}$ & $r_{2}$ & $r_{3}$ & $\mathrm{r}_{4}$ & & & & & \\
\hline $\mathrm{ECE}+\mathrm{EUDC}$ & 16 & 51 & 57 & 19 & 16 & 47 & 53 & 18 & 93.7 \\
\hline FTP72 & 26 & 38 & 53 & 84 & 26 & 37 & 48 & 79 & 94.5 \\
\hline $10-15$ & 23 & 29 & 28 & 36 & 23 & 26 & 28 & 34 & 95.7 \\
\hline $\mathrm{CCDC}$ & 5 & 38 & 73 & 31 & 5 & 37 & 67 & 29 & 93.9 \\
\hline Hybrid & 188 & 299 & 144 & 83 & 187 & 295 & 139 & 82 & 98.5 \\
\hline
\end{tabular}

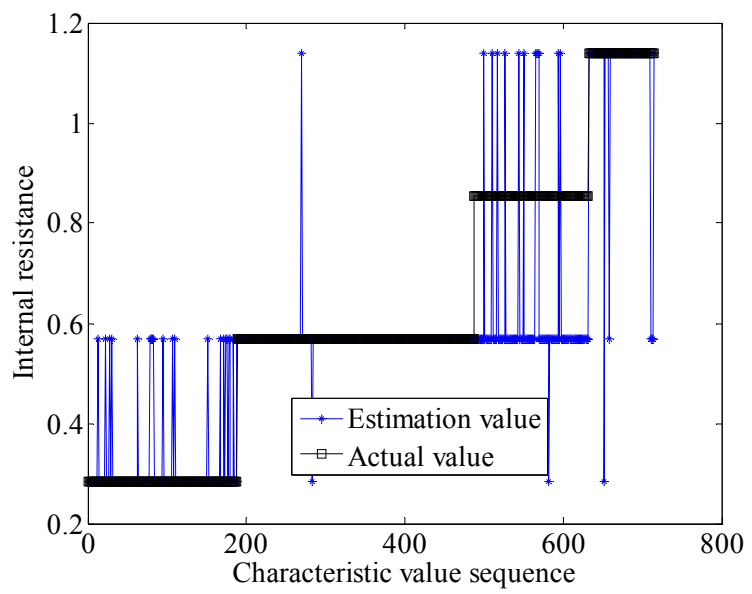

Fig. 16. Estimation results of mixture states under hybrid condition via BP-ANN.

method could achieve values of approximately $98 \%$ compared with the estimation results of the mixture states under hybrid condition in Table III. This finding further proves that the proposed method can estimate battery health states accurately.

After offline training of the HMM, approximately $0.2 \mathrm{~s}$ was taken to recognize a feature vector according to our test. Each feature vector corresponded to 500 data points, namely, $12 \mathrm{~s}$. Therefore, approximately $12.2 \mathrm{~s}$ was taken from the collection of the data to the completion of the estimation. The comparison of this finding with the outcomes in [12] indicated that the proposed method could achieve an online battery test. According to [11], batteries had to be offline to obtain lifetime or the number of cycles, and accelerated test were performed, which took a considerable time. Therefore, online estimation of battery states could achieve a good performance.

\section{CONCLUSION}

This study proposes a method to estimate battery health states based on HMM. Battery life states should be defined according to the internal resistance to estimate battery life. HMM is applied to recognize the life states of the battery.
This study proposes a characteristic value extraction method for HMM. The forward log-likelihood probabilities can be obtained according to HMMs. We can identify the state to which the observation belongs by comparing the probabilities. A recognition framework is built to estimate battery health states. In the experimental stage, the test data of the single and hybrid conditions are built. After preprocessing these data, the characteristic value sequences are inputted in the HMM for estimation. The estimation performance can be evaluated according to the estimation rates. Experimental results shows that the estimation rates of battery life states are all above $90 \%$. This method achieves the purpose of recognizing battery system life states. In addition, the proposed method only needs two parameters and saves a significant amount of time. It also provides a good foundation for online prediction of battery life.

\section{ACKNOWLEDGMENT}

This research is supported by CQ CSTC (CSTC2013yykfC 60005, CSTC2014jcyjA6004, CSTC2013jcsf-jcssX0022).

\section{REFERENCES}

[1] C. C. Chan and Y. S. Wong, "Electric vehicles charge forward," Power and Energy Magazine, Vol. 2, No. 6, pp. 24-33, Dec. 2004.

[2] T. Horiba, "Lithium-ion battery system," in Proc. the IEEE, Vol. 102, No. 6, pp. 936-950, 2014.

[3] P. Bubna, D. Brunner, S. G. Advani, and A. K. Prasad, "Prediction-based optimal power management in a fuel cell/battery plug-in hybrid vehicle," J. Power Sources, Vol. 195, No. 19, pp. 6699-6708, Oct. 2010.

[4] E. Meissner, and G. Richter, "The challenge to the automotive battery industry: the battery has to become an increasingly integrated component within the vehicle electric power system," J. Power Sources, Vol. 144, No. 2, pp. 438-460, Jun. 2005.

[5] J. A. M. Penna and C. L. N. Júnior, "Health monitoring and remaining useful life estimation of lithium-ion aeronautical batteries," in Aerospace Conference. IEEE, 2012, pp. 1-12, Mar. 2012.

[6] Q. Zhang and R. E.White. "Capacity fade analysis of lithium ion cell," J. Power Sources, Vol. 179, No. 2, pp. 793-798, May 2008. 
[7] D. Rakhmatov, S. Vrudhula, and D. A. Wallach, "A model for battery lifetime analysis for organizing applications on a pocket computer," IEEE Trans. Very Large Scale Integr. (VLSI) Syst., Vol. 11, No. 6, pp. 1019-1030, Dec. 2003.

[8] C. H. Piao, Z. Huang, L. Su, and S. Lu, "Research on outlier detection algorithm for evaluation of battery system safety," Adv. Mech Eng., Vol. 6, pp. 1-8, Dec. 2014.

[9] Y. Xie, Z. G. Wang, and Y. S. Zhang, "Development of battery system testing machine," International Journal of Future Engineering, Vol. 2015, pp. 1-7, 2015.

[10] C. H. Piao, Z. G. Wang, J. Cao, W. Zhang, and S. Lu, "Lithium-ion battery cell-balancing algorithm for battery management system based on real-time outlier detection," Math Probl Eng, Vol. 4, pp. 1-13, Apr. 2015.

[11] C. H. Piao, Q. F. Yu, C. X. Duan, L. Su, and Y. Zhang, "Virtual environment modeling for battery management system," J. Electr. Eng. Technol., Vol. 9, No. 8, pp. 1729-1738, Sep. 2014.

[12] T. Parthiban, R. Ravi, and N. Kalaiselvi, "Exploration of artificial neural network [ANN] to predict the electrochemical characteristics of lithium-ion cells," Electrochim. Acta, Vol. 53, No. 4, pp. 1877-1882, Dec. 2007.

[13] B. Y. Liaw, R. G. Jungst, and G. Nagasubramanian, "Modeling capacity fade in lithium-ion cells," J. Power Sources, Vol.140, No.1, pp. 157-161, Jan. 2005.

[14] X. S. Hu, F. C. Sun, Y. Zou, and H. Peng, "Online estimation of an electric vehicle lithium-ion battery using recursive least squares with forgetting," in American Control Conference (ACC), pp. 935-940, Jul.2011.

[15] W. Zhang, C. Cho, J. Liu, and X. Han, "A hybrid parameter identification method based on probability and interval for uncertainty structures". Mechanical Systems and Signal Processing, Vol. 60, pp. 853-865, 2015.

[16] W. Zhang, C. Cho, J. Liu, and X. Han, "A fast bayesian approach for parameter identifications using adaptive densifying approximation technique," Inverse Probl. Sci. Eng, Mar. 2015. (online)

[17] Z. J. Liu, Q. Li, X. H. Liu, and C. D. Mu, "A hybrid LSSVR/HMM-based prognostic approach," Sensors, Vol.13, No.5, pp. 5542-5560, Apr. 2013.

[18] L. R. Rabiner, "A tutorial on hidden Makrov models and selected applications in speech recognition," in Proc. the IEEE, Vol.77, No. 1, pp. 257-286, Feb. 1989.

[19] L. R. Rabiner. "An introduction to hidden Markov models," IEEE ASSP Magazine, Vol. 3, No. 1, pp. 4-16, 1986.

[20] C. H. Piao, W. L. Fu, G. H. Lei, and C. D. Cho, "Online parameter estimation of the Ni-MH batteries based on statistical methods," Energies, Vol. 3, No. 2, pp. 206-215, Feb. 2010

[21] J. Kim, S. Lee, and B. Cho, "Discrimination of battery characteristics using discharging/charging voltage pattern recognition," Energy Conversion Congress and Exposition, IEEE. pp. 20-24, Sep. 2009.

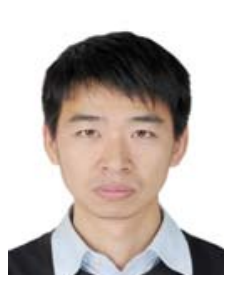

Changhao Piao was born in Yanbian, China in 1978. He is a professor in Chongqing University of Posts and Telecommunication. $\mathrm{He}$ received his B.S. degree from Xi'an Jiaotong University, China in 2004. He obtained his Ph.D. in Inha University, Korea. His primary research interests are new energy vehicle systems, active safety systems, and artificial intelligence.

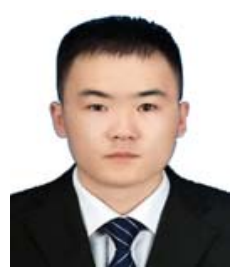

Zuncheng Li was born in Jilin, China in 1989. He is a postgraduate researcher in the Department of Pattern Recognition and Application at Chongqing University of Posts and Telecommunication. His research focus is battery management system and power battery technology.

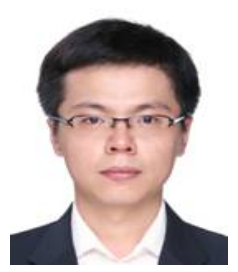

Sheng Lu received his B.S. degree from Xi'an Jiaotong University, China, in 2004. In 2009, he received his M.S. in D.S. from Inha University, Republic of Korea. $\mathrm{He}$ is a professor in Chongqing University of Posts and Telecommunication, China. His interests include automobile electronics, micronano mechanics, and intelligent materials.

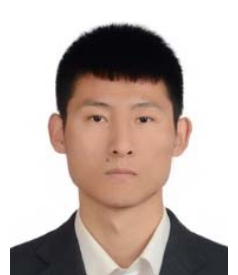

Zekui Jin was born in Gansu, China in 1991. $\mathrm{He}$ is a postgraduate researcher in the Department of Pattern Recognition and Application at Chongqing University of Posts and Telecommunication. His research focus is battery management system and power battery technology.

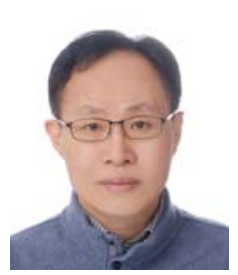

Chongdu Cho received his B.S. and M.S degrees from KAIST in Seoul, Korea, in 1983 and 1985, respectively. He received his $\mathrm{Ph}$.D. from University of Michigan, USA, in 1991. He is currently a professor at the School of Mechanical Engineering, Inha University, Korea. His research interests include composite materials, micronano mechanics, and automobiles. His work has been published in more than 150 publications. 